I AEA

INDC(NDS)-0614

Distr. G+NC

\title{
INDC International Nuclear Data Committee
}

Summary Report of the Consultant's Meeting on

Further Development of EXFOR

IAEA Headquarters, Vienna, Austria

6 - 9 March 2012

Prepared by

D. Brown, Brookhaven National Laboratory, Brookhaven, USA

S. Simakov, Nuclear Data Section, IAEA, Vienna, Austria

April 2012 
Selected INDC documents may be downloaded in electronic form from http://www-nds.iaea.org/reports-new/indc-reports/

or sent as an e-mail attachment. Requests for hardcopy or e-mail transmittal should be directed to services@iaeand.iaea.org or to:

Nuclear Data Section

International Atomic Energy Agency

Vienna International Centre

PO Box 100

1400 Vienna

Austria

Printed by the IAEA in Austria

April 2012 


\section{DISCLAIMER}

This work was prepared as an account of work sponsored by an agency of the United States Government. Neither the United States Government nor any agency thereof, nor any of their employees, nor any of their contractors, subcontractors or their employees, makes any warranty, express or implied, or assumes any legal liability or responsibility for the accuracy, completeness, or any third party's use or the results of such use of any information, apparatus, product, or process disclosed, or represents that its use would not infringe privately owned rights. Reference herein to any specific commercial product, process, or service by trade name, trademark, manufacturer, or otherwise, does not necessarily constitute or imply its endorsement, recommendation, or favoring by the United States Government or any agency thereof or its contractors or subcontractors. The views and opinions of authors expressed herein do not necessarily state or reflect those of the United States Government or any agency thereof.

Notice: This manuscript has been authored by employees of Brookhaven Science Associates, LLC under Contract No. DE-AC02-98CH10886 with the U.S. Department of Energy. The publisher by accepting the manuscript for publication acknowledges that the United States Government retains a non-exclusive, paid-up, irrevocable, world-wide license to publish or reproduce the published form of this manuscript, or allow others to do so, for United States Government purposes. 


\title{
Summary Report of the Consultant's Meeting on Further Development of EXFOR
}

\author{
IAEA Headquarters, Vienna, Austria \\ 6 - 9 March 2012 \\ Summary documentation prepared by \\ D. Brown, Brookhaven National Laboratory, Brookhaven, USA \\ and \\ S. Simakov, Nuclear Data Section, IAEA, Vienna, Austria
}

\begin{abstract}
The Meeting was convened to assess needs and ways for improvement and development of the EXFOR formats to meet future requirements. Participants reported the difficulties of coding complex nuclear reaction and supplemental experimental information in the present EXFOR exchange format, shared their experiences from work with other databases and formats (ENDF/GND, NRDF, ROOT, XSAMS). The general consensus was achieved on the necessity to explore further extensions of the exchange format to facilitate the coding of experimental results, continue development of EXFOR/XML output for easier access and intercommunication with other databases, further development of editors and other software for compilers, establish integrated EXFOR documentation and knowledge database to aid compilers and general users.
\end{abstract}





\section{Table of Contents}

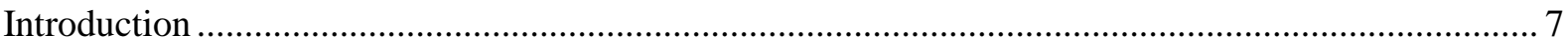

Participants' summaries and recommendations from individual contributions .................................... 7

Developing the XML Schema for Atoms, Molecules, and Solids (XSAMS) ................................. 7

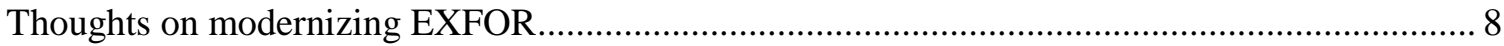

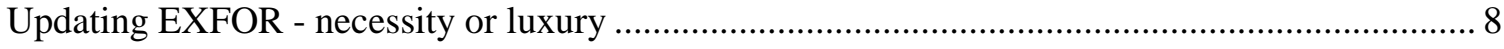

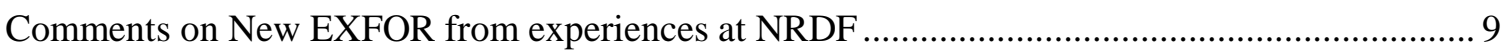

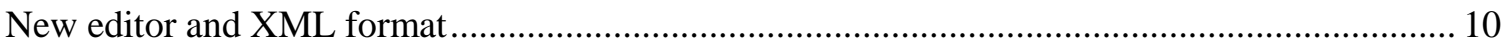

XML in the IAEA-NDS EXFOR-CINDA-ENDF database systems ......................................... 10

EXFOR as a comprehensive source of experimental reaction data............................................. 11



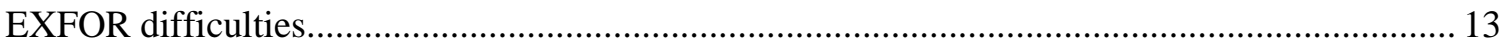

Split coding of the reaction and the measured quantity possible/advantageous? .......................... 15

NDS/IAEA - "EXFOR format: problems of extension and possible solutions ............................... 15

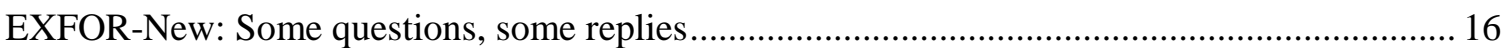

Some thoughts on EXchange FORmat and compilation rules .................................................. 18

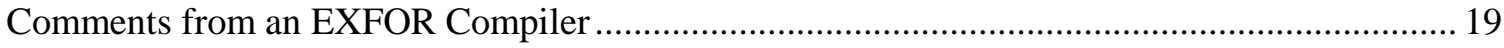

New formats and new features of the NDS EXFOR Web database systems (online-demo) ......... 19



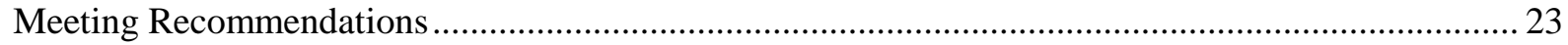

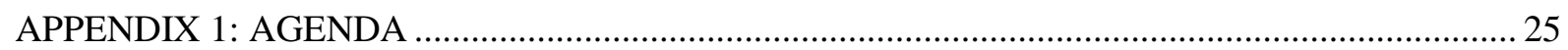

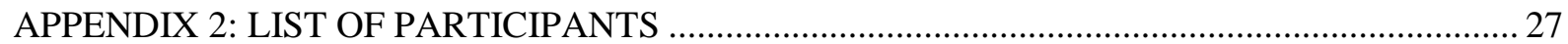





\title{
Introduction
}

The Consultants' Meeting on "Further Development of EXFOR" was held at IAEA Headquarters, Vienna, Austria from 6 to 9 March 2012. Nine consultants M. Aikawa, D. Brown, E. Dupont, S. Hlavac, M. Kato, A. Plompen, V. Pronyaev, O. Schwerer and N. Soppera have attended this Meeting, the IAEA was represented by B. Braams, R. Capote Noy, R. Forrest, N. Otuka, V. Semkova, S. Simakov and V. Zerkin.

This Meeting was organised in accordance with recommendation of the NRDC Technical Meeting held 23-24 May 2011 in Vienna (http://www-nds.iaea.org/publications/indc/item.php?key=indc-nds$\underline{0593}$ ) with a main goal to assess needs and possible ways to develop the EXFOR formats to meet future requirements. Several specific issues were addressed: how to make the EXFOR rather flexible for extensions, universal coding of complex and new type nuclear reactions, review of existing and potentially appropriate formats for exchange, storage and dissemination as well as software related problems.

The Meeting was opened by R. Forrest, Head of Nuclear Data Section of the Department of Nuclear Sciences and Applications of the IAEA by welcoming the participants and explaining the importance of this Consultant's Meeting for the work of the Nuclear Data Section (NDS). The objective and outcomes of the Meeting were outlined by S. Simakov (Scientific Officer of the Meeting). Then participants elected S. Hlavac as a Chairman and D. Brown as a Rapporteur of the Meeting and approved its Agenda (Appendix 1). The list of participants and their affiliations are summarised in Appendix 2.

During two and half days, participants gave presentations (the summaries are collected below) and had intensive discussions. The discussions resulted in the set of consolidated recommendations and conclusions which are collected at the end of this document.

The Nuclear Data Section acknowledged all participants for cooperation and contribution to the Meeting.

\section{Participants' summaries and recommendations from individual contributions}

\author{
Developing the XML Schema for Atoms, Molecules, and Solids (XSAMS) \\ B. Braams, Atomic and Molecular Data Unit within the IAEA Nuclear Data Section, IAEA
}

B. Braams described experience with the development and implementation of XSAMS: the XML Schema for Atoms, Molecules and Solids (http://www-amdis.iaea.org/xml/).

The project that became XSAMS started in 2003 at a meeting of the International Atomic and Molecular Data Centres Network, where a working group was formed to develop an Atomic and Molecular Data Mark-up Language (AMDML). From the start the aim was to code all essential physics of atomic, molecular and plasma-material interaction $(\mathrm{A}+\mathrm{M}+\mathrm{PMI})$ processes into XML. During 2003-2009 the work was coordinated by IAEA and involved a small number of people: Yu. Ralchenko (NIST), D. Schultz (ORNL, now UNT), M.-L. Dubernet, E. Roueff (UPMC Paris, Obs. de Paris), D. Humbert, R. Clark (IAEA); later also P. Loboda, S. Gagarin (VNIITF). The first public release of XSAMS, version 0.1, took place in September 2009 and trial implementations were done around that time for the Atomic Spectra Database (ASD) at NIST, the Spectr-W3 database at VNIITF, the ALADDIN database at IAEA and BASECOL at the Paris Observatory.

Efforts to implement XSAMS expanded greatly when the schema was adopted by the Virtual Atomic and Molecular Data Centre (VAMDC; www.vamdc.eu), a European Union Framework-7 project that brings together 21 partners ( +3 external), primarily from astrophysics and astronomy, that are together responsible for 19 relevant databases. The project has about 3M Euro in funding for the period Jul 2009 - Dec 2012. 
VAMDC was created in order implement a common interface and enable single queries across multiple databases and facilitate data publishing and quality control. The development of XSAMS beyond version 0.1 was led by VAMDC 2010-present, and XSAMS-1.0 was adopted in Feb 2012. The complete VAMDC-XSAMS schema may be found via http://www.vamdc.eu/documents/standards/ .

In March 2012 the implementation status is that all VAMDC partner databases now offer XSAMS query and output options and access via the "VAMDC SQL Subset 2" (VSS2) query language. However, on all databases the XSAMS and VSS2 options are still for developers only; proper user experience is left for Q2-Q4 2012.

Some considerations for EXFOR based on the XSAMS experience have been offered. XSAMS is an effort to encode all essential A+M physics. The theoretical basis is the many-electron Schrödinger equation with known interactions, and generally the Hartree-Fock picture is available to classify states. It all works best for atoms and is more difficult for molecules. Nuclear physics has not such a clear theoretical basis and the classification of processes can probably not be as detailed as is done for atoms in XSAMS. Also XSAMS was designed for interoperability among many different databases that were quite separate originally; in the case of EXFOR one has a single database in a more tightly organized community. Finally, the XSAMS implementation experience was in all cases very valuable for database developers as it forces precise specifications and helps to find errors. On the other hand, end user experience is still lacking in early 2012.

\section{Thoughts on modernizing EXFOR}

\section{Brown, NNDC, Brookhaven, USA}

D. Brown presented several reasons for modernizing EXFOR (+ENDF) data formats, commenting that many issues are common to both library formats. Chief among the technical reasons given were interoperability, extensibility and ease of use in new code packages. The ease of use should not be overlooked because of the need to attract new members to the field of nuclear data and a complex data infrastructure can turn off any new users.

He then introduced the Generalized Nuclear Data (GND) format, a hierarchical (XML) format for evaluated reaction data and transport data. He also introduced the Fudge package for manipulating, translating and processing GND data. Both Fudge and GND are open source and mostly use off-theshelf tools (xml validators, Python, matplotlib, numpy). The GND format can support evaluated reaction data and either Monte Carlo or deterministic transport data. Support is planned for nuclear structure data (i.e. ENSDF) and experimental reaction data (i.e. EXFOR).

The GND/Fudge project has been proceeding for roughly 3 years at the 1.5 FTE level. The work is strongly leveraged with support from the US National Nuclear Security Administration's Advanced Strategic Computing program (for modernizing the data processing codes), but most of the work went into coding tools to work with ENDF and this funding came from the American Recovery and Reinvestment Act of 2009.

The experience in transitioning the evaluated data libraries from the ENDF format to the GND format will inform any potential transition from the EXFOR format to any new format. In particular, CSEWG anticipates a transition period of as much as a decade in which ENDF libraries will be released in both new and old formats.

D. Brown also presented several concepts common to ENDF and EXFOR where common features could be encapsulated in XML and shared. He agrees with V. Zerkin's assertion that the EXFOR data hierarchy is solid and probably not in need of revision. However, the low-level data structures in EXFOR and ENDF could easily be consolidated into a single format. He also presented some syntax which could replace/supplement EXFOR REACTION fields.

\section{Updating EXFOR - necessity or luxury}

\section{S. Hlavac, Institute of Physics, SAS, Bratislava, Slovakia}

EXFOR has been in use for more than 40 years. It was designed to exchange data on neutron induced reactions, relevant for applications, between nuclear data centres. In low energy neutron induced reactions only selected data types were collected and coded. The situation changed, when charged particle induced reactions started to be compiled in EXFOR. The variety of data types and the 
complexity of reactions increased dramatically and because of this the coding of new data types has become difficult. New data types for neutron induced reactions and covariances are also difficult to include in the existing system.

The rigid format also represents a limitation, this is a consequence of the information technology available in the sixties. The EXFOR user community has changed too, from nuclear data experts to a more academic community, which now produces the majority of charged particle data, and possibly even the majority of all new nuclear data in EXFOR.

If the EXFOR system is to serve all potential future users, it needs some modernisation, mainly because of new types of data (covariances, complicated reactions ...), changing user community and new available information technology.

Because there are now practically no limitations from available information technology, many limitations of existing system may be dropped as for instance:

1. limitation to 80 columns,

2. allow for flexible free format input,

3. full text description whenever possible (institute, method, detector, ...),

4. reaction coding close to standard usage in the literature,

5. standard units without special/multiple abbreviations,

6. allow for covariance coding and storage as well as future expansion.

It may be useful to replace the EXFOR text format with an explicit hierarchical format, which should already have supported infrastructure and be of high performance. High energy and experimental nuclear physics have a ready-made solution: ROOT. One of the solutions to modernise EXFOR may be to develop an EXFOR application based on the ROOT framework.

Data in ROOT is stored as a binary hierarchy (similar to HDF5) and is self describing so one can deserialize data simply and data in each file are automatically indexed for quick random access. ROOT uses $\mathrm{C}++$ but there are Python (pyROOT) and Ruby wrappers.

ROOT is a complicated framework, but many of its aspects would be useful: huge data storage, fast access to data, flexibility and ease of extension and advanced infrastructure. A system based on ROOT may bring another advantage for user community, because many experiments nowadays use ROOT for the analysis of data, there are already advanced users of ROOT in the nuclear data field. ROOT drawbacks represent mainly migration to the new system and the need to acquire the necessary expertise in using $\mathrm{C}++$.

\section{Comments on New EXFOR from experiences at NRDF \\ K. Kato, Hokkaido University, Japan}

K. Kato presented the JCPRG database NRDF and explained its characteristics compared to EXFOR. The basic idea of NRDF was developed by H. Tanaka et al. in the early 1970's as self-describing database. To realize this idea for charged-particle induced nuclear reaction data, NRDF has great flexibility in description of not only experimental data but also experimental processes including experimental conditions, devices and methods by using an XML like format and free text.

In the presentation he mentioned several points that should be addressed in a successor to EXFOR. In NRDF the data sets are:

- BIB, EXP or Data, all values are in a key-value pair like XML. Therefore it is very easy to add new data items describing new experimental techniques, new accelerators, new detectors, new observables etc.;

- as free text are given the various data of very complex parts such as equations and the parameters can be easily included;

- even new tags can be defined since all the tags have a description printed with them in a comment. 
NRDF started as a charged particle database but now includes photonuclear and reaction data suitable for medical application. As examples of recent data, there is need to include information on broadening/resolution effects for data and to include hypernuclei, $\pi^{0}, \pi^{+}$and $\pi^{-}$projectiles (to support the Japanese hypernucleus program).

Since NRDF is a superset of EXFOR and the mapping is not one-to-one, the original translator of compilation data from NRDF to EXFOR didn't was not completely successful. To solve this problem JCPRG developed HENDEL (Aikawa's talk) which is a compilation system for data of both NRDF and EXFOR.

The project to make a new NRDF system started on the basis of XML which is a desirable scheme to describe various kinds of retrieval systems and other programs in a web-based system.

\section{New editor and XML format \\ M. Aikawa, Hokkaido University, Japan}

M. Aikawa presented a talk about the HENDEL editor and the GSYS digitizer, which are software constructed in JCPRG. The HENDEL editor is a web-based data input system. It is available on the website (http://www.jcprg.org/hendel/) but restricted by ID and password at present. Its advantages are 1) a standard web form interface, 2) easy and obvious to use, and 3) simultaneous output in both EXFOR and NRDF, which is the JCPRG original database.

When compilers use the editor, they can avoid studying formats and codes of EXFOR and NRDF beforehand. Once data were saved in the editor, they can immediately look at, check and modify it. The editor is successful in reducing mistakes in terms of codes and formats. The number of JCPRG compilations since its construction in 2001 has grown dramatically to over 800 entries so far.

On the contrary, there still remains a difficulty in authors' proofreading in the JCPRG compilation process. Neither EXFOR nor NRDF formats are easy for authors to understand and confirm the contents. It is necessary for the process to create a more human-readable format, for example, a format using understandable XML tags. JCPRG has a plan to create a new editor using the new format and an open web-based environment "WEBBLE" (http://cow.meme.hokudai.ac.jp/WebbleWorldPortal/) under a collaboration with Meme Media Laboratory in Hokkaido University.

\section{XML in the IAEA-NDS EXFOR-CINDA-ENDF database systems} V. Zerkin,NDS, IAEA, Austria

\section{Topics}

1. Using XML for loading databases in NDS systems (since 2002)

- My XML-SQL for loading databases: EXFOR, CINDA, ENDF

- EXFOR relational database: schema and its reflection in my XML-SQL

2. EXFOR for end-users (output from NDS system)

3. EXFOR-XML (2009)

- Goal and basic principles

- Technical details and implementation

- Translation to an interactive "super-fancy" Html using language XSL

- Release on Web (2011)

- Advantages, disadvantages and alternatives to XML

4. Software - deciding factor for migration from EXFOR to XML

5. EXFOR "Standard" output

- Problems with EXFOR files for end-users

- EXFOR "Standard" output: goals, audience

- Basic principles: no pointers, no common blocks, no dictionaries, sorted columns, identification for automatic processing

- EXFOR "Standard" output into Text and XML files 
$\underline{\text { Summary }}$

1. "My XML-SQL". Here XML language is used as 'a form' for transferring data from formatted text to a relational database. The main goal is to populate and update relational databases. Using XML as an intermediate format in the chain "EXFOR/ENDF/CINDA $\rightarrow \mathrm{C}$ programs $\rightarrow$ XML $\rightarrow$ Java program $\rightarrow$ SQL $\rightarrow$ Relational database" is just convenient and rational from the practical view point to make fast, universal, multi-platform database/software system with limited man power in a short time.

2. EXFOR was originally design as an EXchange FORmat between nuclear data centers - it was not intended to be directly used by end-users (it is not really suitable for them). Nuclear data centers try to disseminate experimental data in the forms more convenient for end-users than original EXFOR, namely: as various output formats, tables, plots. This is defined by users' needs, requires software development and not always easy to achieve. NDS Web system offers number of output options, including variations of EXFOR-like formats with information from dictionaries: EXFOR interpreted (X4+), EXFOR interactive tree (X4 $)$ ); computational formats (C4, C5, Table) and plots. Since November-2011, NDS Web system provides also two types of XML output formats.

3. EXFOR/XML was developed as output from NDS EXFOR system in 2009 as a pilot project. Structure of this XML format is full equivalent to EXFOR structure. This allows reconstructing initial EXFOR file from its XML presentation. It also includes information from dictionaries (meaning of codes) and interpretation of coded parameters. NDS Web system also provides interpretation to interactive HTML/CSS/JavaScript using XSL

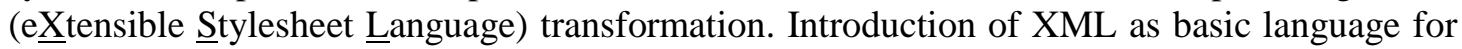
EXFOR could have advantages and disadvantages; practical purpose and price of full implementation of such a project should be well understood before further development.

4. EXFOR/XML (as well as original EXFOR) has a structure oriented to the logic of compilation process, therefore end-user reading such files has to know not only information from the dictionary system, but also to understand structure and links within the EXFOR file, such as: common data, pointers, rules. It is difficult and time consuming; therefore for end-users a "Standard" output from EXFOR system has to be provided. The format proposed as "Standard" output is much simpler then EXFOR with: no pointers, no need in dictionaries, no common blocks, sorted columns, etc. Software generating output in this format is ready, the data in two forms (text and XML files) has been offered by NDS Web system since 2011.

\section{General conclusion}

1. Software infrastructure for producing XML on the current EXFOR exists. It can be used to convert EXFOR + Dictionaries to any other formats (including, probably, ROOT)

2. Since 2011 two types of XML presentations of EXFOR data are available on the IAEA NDS Web site

3. EXFOR XML Input and Output serve different purposes and are oriented to different users' communities

4. There is not enough experience in using of existing EXFOR XML: both EXFOR XML formats have still draft status. (No feedback from potential users yet; questionable subject)

5. Technically migration of EXFOR to EXFOR-XML can be done, but with large investment in the software development.

\section{EXFOR as a comprehensive source of experimental reaction data}

\section{A. Plompen, European Commission, DG Joint Research Centre, Nuclear Physics Unit, Institute for Reference Materials and Measurements, Belgium}

EXFOR as an experimental nuclear reaction data library is unique. It has public access and is the basis for any high quality and comprehensive nuclear data library. In particular, it serves the needs of evaluators and validators of the nuclear data libraries used by authorities, industry, engineers, scientists and students active in important fields of societal interest: safety, security and innovation of nuclear energy, nuclear waste, medicine, materials and components research, nuclear science. 
Improved evaluations and systematic validation would not be possible without a comprehensive database of results from experiments. The database is the world-wide recognised tool for knowledge preservation in the field of nuclear reaction experiments and allows to capitalise on and save many new investments in experiments and manpower. A harmonised approach to this database and high standards for its contents are of the utmost importance for this "Mother of all nuclear reaction libraries".

The conception of EXFOR lies far in the past and despite the great foresight of its inventors and developers, new demands and impressive software developments in the past decade call for a careful evaluation of the status of EXFOR and the services provided with it. These services include the services offered to the users and the services offered to the compilers and maintainers of the database.

The following recommendations are made:

1) Governance. For such an important database it is essential that a proper set of rules and goals are laid down that are agreed by the international community and in particular the Nuclear Reaction Data Centres. A strategy document is required that establishes which user groups the library should serve and which services should be maintained and developed. Purpose, scope, quality, validation, interfaces and user-orientation are important keywords to be addressed in such a document.

Feedback from users is now a weak point and a method for a systematic approach to establishing users interest and needs should be established (e.g. through an EXFOR user group that periodically reviews the services that are offered and providing recommendations for improvements and new directions).

2) Accurate evaluations require considerable detail about experiments in EXFOR entries. The Nuclear Physics Unit has a clear opinion about this and materialised in the form of a template, adopted by NRDC, specifying what should be detailed for time-of-flight experiments. Similar level of detail is needed for other measurements, e.g. activation data. Data required to interpret the data reported are often essential for a re-evaluation: resolution functions, neutron spectra, detector models. These may go into the database itself or be documented in an archive of contributed documents.

3) Accurate evaluations require proper uncertainties and covariances from experimental data. These should be readily obtained from EXFOR. The use of the "Guide for the expression of uncertainty in measurement" (JCGM 100:2008, www.bipm.org (2008), abbreviated as GUM) should be promoted. It is general, applicable to all fields of measurement, precise and concrete. Important organisations support it and the guide is written in their name: BIPM, IEC, IFCC, ISO, IUPAC, IUPAP, OIML. Of course the GUM leans heavily on first order uncertainty propagation and does not warrant against situations where this is not applicable. However, in combination with detailed reporting of input quantities and measurement and analysis expressions these situations are adequately covered. In particular, we should adopt and internalize the uncertainty concept of that document, speak about knowable quantities only, correlated/uncorrelated uncertainties, make use of TYPE A and TYPE B qualifications (the latter can be considered default).

4) It is important to have mechanisms for correcting data, either because data rely on standards that may evolve, or because of corrections and correction factors by evolving understanding about the experiment. Links between data sets are important in order to establish the proper weight of a given data set in an evaluation.

5) As an EXFOR user one should find what one needs a) always, b) easily, c) in the form of choice. High standards should be maintained for the web-interface. This is what the new generation is used to and expects from a highly developed field. Hard to understand features should be avoided, flaws undermine confidence. One should not need be an expert of the database to navigate the web interface. A google-like search feature would be a dramatic step forward. A modern look to the page would also help (easy look, easy overview, collapse/expand features). 
6) Avoid overlap with other databases (ICSBEP, IRRPHE, SINBAD, ...)

7) Nuclear reaction data embedded in special purpose files should also be found in EXFOR.

\section{Coding of complex reactions in EXFOR \\ V. Pronyaev, Nuclear Data Centre, Institute of Physics and Power Engineering, Russia}

The problems of complex reaction's coding (REACTION string, sub-fields SF5 to SF7) were discussed. The complex reactions are considered as important for including for compilation in EXFOR are new results obtained in the multi-detector, multiparametric measurements and measurements in coincidences. There are many such data obtained in different classical areas of interest such as primary and secondary fission product yields, their kinetic energies in coincidence with yields of prompt fission neutrons. The other examples are reactions of light nuclei with sequential emission and breakup reaction mechanisms, which, using known kinematics and position of the detector can be experimentally separated (such as ${ }^{9} \mathrm{Be}+\mathrm{n}$ ). Many charged particle reactions in the medium and high energy domain belong to the complex reaction category. With current development of the multiparameter and multi-coincidence measurement technique ( $4 \pi$ multi-detector systems, etc.) more and more results will appear. They are extremely important for evaluators because they allow to test and adjust parameters of nuclear models used in evaluation.

The present structure of EXFOR has limited opportunity for coding of complex reactions. It uses a rather archaic system of codes in SF5 to SF7 dictionaries and their combinations, with no unique definition of the reaction type, and requires consensus between all centers when new code is introduced. Many complex reactions can not be compiled in EXFOR at present.

A new scheme of the complex data coding is proposed. It is based on a detailed description of the reaction string, including particles registered or intermediate (when they are known from conservation laws and kinematics of the reaction), observables registered in the experiment and the correlation/coincidences between the observables. The observables which can be now included are: particles and nuclides, states, spins, energies, angles. This extended REACTION coding scheme is much close to how the experiment was set-up.

Several examples of the coding are shown.

There are several advantages comparing to the existing reaction codings: it allows to compile results of any multi-parameter, multi-coincidence measurements of any complexity of data without the long procedure of approval of a new REACTION code, because all combinations are in the "dictionary" by default; the reaction description is fully computerised, there is nothing concerning the reaction and the reaction conditions that has to be given in free text.

There are the following drawbacks in comparison with the existing EXFOR reaction coding: (i) problems in „human-readable“ presentation as ASCII text files, in full detail; (ii) reactions, which are given in existing EXFOR format as the same (but measured using different observables in the experiment) have different reaction string.

\section{EXFOR difficulties}

\section{S. Simakov, NDS, IAEA, Austria}

EXFOR users are usually satisfied with a descriptive textual information and conventional abbreviations given under the keywords like REFERENCE, INSTITUTE, COMMENTS, SAMPLE in the EXFOR Entries. However essential difficulties arise when reading factual information such as a description of nuclear reaction (coded as Reaction String) and measured or deduced physical quantities (reported in the DATA and COMMON blocks).

\section{The specific inconveniences for users of reading output formats $\mathrm{X4+}, \mathrm{X} 4 \pm$ (that reflect the structure of exchange format $\mathrm{X4}$ ):}

- reaction string is restricted to 55 characters and has a (FORTRAN) fixed format $A(a, b) B, \mathrm{SF} 5, \mathrm{SF} 6, \mathrm{SF} 7, \mathrm{SF} 8, \mathrm{SF} 9$. The information allowed in each of these subfields is one char variable (coding word). These codings and their combinations attempt to represent an increasing 
diversity of experiments that is implemented through the EXFOR dictionaries. The most complicated one is \#236 which allows as many as 1118 combinations in subfields 5 to8. The codings have short names and are not self-explanatory, the descriptions are given not for all of them.

- COMMON and DATA blocks are separate parts of the Entry for storage of measured or derived quantities. Physics/quantities are encrypted as EXFOR specific codes and their combinations (e.g., PAR, DA/CRL, D/A, REL, EN-SEC ...). This maybe convenient for experienced compilers but not for a user who has to read the original paper to understand the content of Entry.

\section{Possible modernization of exchange format X4:}

- principally, differentiation between (i) observables - what is already well known or is detected/fixed in the experiment like target, incident particle, detected ejectiles or products including energy, angle, combinations among them (coincidence) etc. - they are well defined by physics, have conventional abbreviations, are easily coded; and (ii) derived physical quantities what was reported in the paper as quantitative characteristics of the reaction proceeding via pathway specified in the experiment - they are derived from detector counts, processed and corrected to report a physical quantity which is independent of the measuring procedure and may have different representations depending on the author preference.

- technically, replacement of the present A(a,b)B,SF5,..,SF9 string and COMMON/DATA blocks by one (or two) block(s): reaction string/block (e.g. A $\{a t r\}(\mathrm{a}\{a t r\}) \mathrm{C}\{a t r\}(\mathrm{b}\{a t r\}) \mathrm{B}\{a t r\})$ and Quantities or DATA (grouped in classes according to the physical meaning, e.g. SIGMA $\{a t r\}$, RESONANCE $\{a t r\}$, LEVEL_DENSITY etc.). Usage of XML technology with flexible format and option to describe the physical variable via a set of attributes (atr is pair of named parameter and its value) looks to be a possible approach.

This may result in more flexibility for compilation and more understanding for end-users of the coding of Reaction and Quantities, since it allows:

(i) more natural (accepted in physics) syntax for exclusive $\mathrm{A}(\mathrm{a}, \mathrm{xb}) \mathrm{C}$, compound $\mathrm{A}+\mathrm{a} \rightarrow \mathrm{C} \rightarrow \mathrm{B}+\mathrm{b}$ and other complex reactions;

(ii) specification of all parameters relevant for physical variables in one logical place via attributes (e.g., Incident_Particle $\{$ type, energy, spin, ... $\}$ ) or their experimentally fixed combination (Correlation $\{$ ejectile1 $\{$ energy, angle,..\}, ejectile $2\{\ldots\}$, compound $\{$ resonance, spin,...\}, ... $\}$ ) that fully specify reaction pathway and its unique probability;

(iii) long named and human understandable designations for quantities instead of short abbreviations or code words;

(iv) introduction of new attributes for testing purpose without crashing checking codes;

(v) inter-communication with other databases as soon as they use XML as well.

Proposals for new coding were given for ${ }^{12} \mathrm{C}\left({ }^{14} \mathrm{~N}, \mathrm{~d}\right)^{24} \mathrm{Mg}^{*}(\alpha){ }^{20} \mathrm{Ne}$ (g.s.) reaction (by comparing with existing Entry T0117003) and for resonance parameters derived from (n, $\gamma)$ (Entry 12633002). The number of Entries in EXFOR reporting the data measured by coincidence technique is estimated as $\sim 1500$ or $\sim 7 \%$. The number of Entries where multiple reaction string formalism is used to define the resonance parameters probably is of the same order.

While modernization of exchange format has to be elaborated and tested before real implementation, EXFOR could be improved without affecting of current X4 format through: implementation of detailed (textual, graphical ...) explanations for coding words using LEXFOR fragments, extending of EXFOR line beyond 66 symbols, modernization of Editors and checking codes.

The neutron spectra compilation in EXFOR was reported as another example of difficulties. In this case they are of organizational nature (a need to agree with NRDC a new INC-SPECT dictionary) and of technical ones (attempt to keep spectra as separated Entries forces the use of nuclear reaction string for facility description, that has no sense for, e.g. reactor as a neutron source). The XML-like structure of INC-Source (instead of current two INC-SOURCE and INC-SPECT) enables the option to describe the used facility, energy spectra via attributes or include links to other Entries and databases. 


\section{Split coding of the reaction and the measured quantity possible/advantageous?}

V. Semkova, NDS, IAEA, Austria

EXFOR files contain key information for the experiments in a compact and concise form. Try to keep the file as short as possible so users can get to what they want without wading through text.

Templates for different types of experiments would be useful during compilation in order to include all important information for the experiment in EXFOR and even request information from authors if it is not available in the article.

Currently, EXFOR can be presented in several different output formats as generated by V. Zerkin's comprehensive web system.

Searching experience could be improved. SF fields are helpful for breaking degeneracy in search, but they are not at all obvious to compilers or users.

Restrictions in the length of the reaction/quantity information leads to coding that can be difficult to interpret. Perhaps HTML or X \pm should be set as the default output.

There are number of cases were the same codes have different meanings when placed in different SF fields. The SF fields can easily be jumbled if the wrong number of commas is used in a REACTION code. This may lead to incorrect interpretation of the reaction/quantity information.

\section{NDS/IAEA - "EXFOR format: problems of extension and possible solutions}

\section{Zerkin, NDS, IAEA, Austria}

\section{$\underline{\text { Topics }}$}

1. What is EXFOR nowadays: format, library, database, retrieval systems, codes

2. What we are going to discuss: input, output formats or both

3. Some inconvenient questions to EXFOR format (issues in last 13 years)

4. Structure of EXFOR file and logic of compilation

5. Coding of information in EXFOR: concept of Keywords and Codes, nesting and meaning of parameters

6. Possible options of using XML as language for EXFOR

- extension of existing EXFOR by named parameters (generalized attributes)

- redesign EXFOR using XML language

7. Input/output of bibliographic information in NDS EXFOR system. Using external data sources: NSR, BibTex, DOI lists, etc.

8. Coding of complex Reactions

9. Attributes of particles (and other SF-subfields)

- Multi-step reactions

- Attributes of particles (and other SF-subfields)

- Pre-EXFOR (adding information to an extended EXFOR, with automatic conversion to official EXFOR)

10. Possible coding of multistep reactions using XML

11. About "unfixable" problems in EXFOR

\section{Summary}

1. Until now, most of the issues, regularly appearing in EXFOR system due to the needs of essential extensions, were solved within or outside existing format and dictionary system in a satisfactory manner.

2. EXFOR structure essentially corresponds to the logic of published experimental paper, and therefore it does not need any revision.

3. There are at least two ways to solve the problem of a description of complex reactions: (a) by extending of existing reaction specifications in EXFOR using patches and (b) by redesign of EXFOR coding of reactions using XML language. First solution will solve problem partially but with lower cost. Second is universal solution, but needs lots of effort to solve all problems associated with EXFOR software. There is not an urgent need to completely redesign the 
reaction specification having in mind the limited number of exotic and very complex reactions.

4. In order to resolve the currently existing issues with dictionary system, extensions of the format and effective compilation, more attention should be paid to EXFOR utilities, such as: checking code (which is very old and difficult to extend), editor, and retrieval software. Plans of software development (functionality, technology) should be seriously considered by the NRDC community. In order to foresee the consequences of a serious format modification, NRDC has to consider the existing software which is dependent on the EXFOR format and try to consult with software developers and maintainers.

$\underline{Q \& A}$

1. Do we have really "unfixable" problems in EXFOR?

No.

2. Can we avoid "XML revolution"?

3. Do we have [qualified] manpower for complete migration to XML?

Yes.

Not yet.

\section{Concluding remarks}

1. EXFOR has some internal reserves for extension without "XML revolution"

2. Revision of the Dictionary system is needed

3. Some problems can be solved using outside sources (NSR, PDF, BibTex)

4. XML should be discussed more to find solutions for an extended coding of reactions

\section{EXFOR-New: Some questions, some replies}

\section{O. Schwerer, Vienna, Austria}

There are three main reasons to consider a renewal of the EXFOR format: to make life easier for compilers, to improve the user-friendliness for the end-users, and to better accomodate new or complex data types. All of these are legitimate, but it is good to be clear which of these issues a particular proposal is supposed to address.

Many of the issues in discussion can be solved within the present system, by:

- Software (EXFOR editor, check programs, output formatting programs, and others)

- Updating/restructuring dictionaries

- „Soft“ updates of the fomat (e.g new keywords)

- Changing NRDC policies (allow non-experimental data, introduce quality flags)

Reforming the format will not automatically solve most problems of compilation. Manuals such as the LEXFOR manual will still be needed, as it describes not mainly format questions but definitions of quantities and their related independent variables. This will be needed independently of the format.

Most inconveniences for compilers can be taken care of by the (possibly further improved) EXFOR editor. The most time-consuming part of compilation is often reading the article and understanding what was measured, which is independent of the format.

User-friendliness is totally an issue of output formats, of which a variety is already available, ranging from various computational formats to human-readable formats for various user groups.

The Exchange Format was developed only for exchange between data centres, and relatively sophisticated user formats - printing nice tables and explaining all the cryptic abbreviations - existed already in the 1970s.

For the compilation of new and complex data types certainly an upgrade of the present REACTION formalism is needed. Various options are being explored. One possibility would be to introduce a new keyword which could be used alternatively to REACTION in case of complex data types.

The other question is where the limit of introducing ever more complex data types should be - they require considerable efforts in extending and maintaining the manuals and dictionaries, while the actual user needs are not always clear. Guidance by the governing bodies of the NRDC network in this matter should be sought. 
Some of the perceived problems, as presented at this meeting, are already solved or can easily be solved within the present system. Examples are:

- Addition of new dictionaries and new keywords: is a standard procedure

- Inclusion of ratio to standard when not given explicitly by author: implemented (but so far rarely used)

- Inclusion of non-experimental data: EXFOR contains e.g. some evaluated charged-particle data

- Thick manuals or the full dictionaries should be needed only by compilers; for users it should normally be sufficient to use a human-readable output format and/or the „EXFOR Basics“ Manual, or a computational format

- For the (occasionally cryptic) keywords (maximum length is 10) there exists a selfexplanatory „long version“ in dictionary 2 which could easily be used in output formats.

\section{An idea on the quantities dictionary 236}

One of the problems of the quantities dictionary 236 comes from the fact that it contains only the REACTION subfields SF5 - SF8 which define the quantity (excluding subfields SF1 - SF4 which describe the reaction). While this is useful, because the majority of quantities can occur with many or almost all reactions, it leads to problems with quantities which are meaningful only for a very restricted set of reactions such as fission, or which become a special case when they are used with variable products such as ELEM/MASS.

These problems may concern various areas, including understandability for compilers, the best wording for the expansion (full text explanation) of the quantity in the dictionary, and for the check program CHEX.

Therefore it may be worth considering to introduce optional flags for a link to certain values of REACTION subfields SF2, SF3, and SF4.

E.g., certain quantities would only be valid for SF3 = F (fission). This could be verified by the check program CHEX, and the expansion text in the dictionary could be more specific.

Similar considerations could apply to certain values of SF4 (the flag would probably be used mainly for variable SF4 such as ELEM and/or MASS), and SF2 (e.g. for quantities for reactions with projectile $=0$ ).

\section{A proposal on the documentation: „EXFOR Knowledgebase“}

A problem with the EXFOR documentation is that information on the same or related topics is distributed over several documents. The reason for it is that that the various documents are meant for distinct groups of readers (compilers / software developers / data centre managers, etc.), but the topics and the needs of these user groups are overlapping, and it is sometimes difficult to locate the needed information.

Therefore we propose to integrate the information available in various existing sources, by introducing a common indexing or keywording system. The user would input the appropriate keyword and the software would provide a list of links to suitable pages in one or more of the documents.

The following source documents should be included:

- EXFOR Formats Manual

- LEXFOR Manual

- EXFOR Basics Manual

- Dictionary Manual

- Dictionaries in full, including explanatory text given there

- Protocol

- Network document

Possibly (or in a second step), also recent CP and 4C memos could be included. 


\section{Some thoughts on EXchange FORmat and compilation rules E. Dupont and N. Soppera, NEA Data Bank, OECD, France}

EXFOR was meant as an exchange format between Nuclear Reaction Data Centres (NRDC), which may store the contents under any system and provide output in any "format" (plots, text, table, hyperlink text...).

There are obvious limitations in the EXchange FORmat and in dictionary format. For example, several rules of EXFOR are puzzling for humans: fixed width/fixed number of columns; text or parentheses in fixed column number 11 or 12; wrapping of data lines; condensed coding that require dictionaries; redundant control codes; difficulty to add external information (covariance, neutron-sources). Some limitations apply also to computers: limited character set; limited number of digits. The latter limitation is an actual issue for the precision of Time-of-Flight data and to preserve the mathematical properties of covariance matrices.

However, most of these limitations could be avoided by improving compilation rules and dictionaries. For example, the use of EXFOR editors enforces format and consistency. In addition, editors free us from most format limitations and allow changing format without hurting compilers.

Another possible improvement lies in the simplification and homogenisation of dictionaries. There are too many; some are deprecated some are not and it is not always obvious. In addition, when distributing dictionaries to other Data Centres: one (exchange) format should be enough (3 different versions at present). The generalisation of the use of wildcards in Dict.236 is another example of possible simplification. Finally, the dictionaries should be updated together with the EXFOR master for obvious consistency reasons.

Evolution of the current EXchange FORmat is possible and is done regularly within the NRDC framework. In particular, format extensions to add new keywords for source spectra, covariance data or bibliographical resources is possible and suppression of obsolete parts is possible too (e.g. control codes, useless dictionaries). However, dramatic evolutions may call for more drastic format change.

Indeed, revolution might be more appropriate if we want to modify the structure of the EXchange FORmat. Defining a new format from scratch is probably not realistic today. However, the ongoing development in the US of a Generalised Nuclear Data (GND) format for evaluated data is a good opportunity for NRDC to study its adoption as a new exchange format for experimental data. Nevertheless, the international protocol to develop and maintain this new format remains to be defined.

The benefits of XML-based formats (such as the GND format) were already listed on Brown's slides: One can mention more specifically the flexibility in layout (e.g. comments, line lengths); the possibility to enforce a basic validation using schemas (XSD); the possibility to use standard software and to more easily connect to other XML-based formats (e.g. GND, XSAMS). In addition, a new XML-based format would remove all obvious EXchange FORmat limitations listed above.

As for the drawbacks, XML-based formats can be more verbose with large amounts of data (e.g. tables); the size of the files usually increases; XML technologies can be complex (for physicists).

Of course, this new format cannot directly address complex reaction coding or NRDC administration issues. Nevertheless, XML-based formats certainly improve the interoperability between heterogeneous systems (and a fortiori between all XML-based formats) used by compilers, Data Centres, advanced users (including a new generation of users).

However, adopting a new exchange format between Data Centres would need a lot of preparation and it is important to stress that a literal translation from the current EXchange FORmat to some XMLbased format is certainly not enough to justify the effort. First, we need to assess the resources and manpower needed to (1) upgrade our compilation tools (EXFOR editors, checkers); (2) convert the current EXFOR Master and Dictionaries; (3) update the documentation; (4) prepare the simultaneous adoption by all Data Centres of a new exchange format.

Finally, we would like to summarize our contribution in the following recommendations:

- the usage of EXFOR editors should be highly recommended and their development further supported;

- the evolution of the current EXchange FORmat is still possible;

- the development of a new exchange format will require more discussions, tests and careful preparation before simultaneous adoption by all Data Centres. 
- the Generalized Nuclear Data (GND) format developed in the US is a good basis for a new exchange format and its capability to describe EXFOR reactions and to handle the variety of EXFOR data tables should be assessed by Data Centres in close collaboration with GND developers.

\section{Comments from an EXFOR Compiler}

\section{N. Otuka, NDS, IAEA, Austria}

Distinction between EXFOR Exchange Format and EXFOR Output Formats is reviewed briefly based on the description in the EXFOR Formats Manual (IAEA-NDS-207). It is stressed that the Exchange Format is specialised for inputs by compilers, and not necessary to be customised for end-users. Output formats must be further developed to meet needs of EXFOR users from various fields, while the Exchange Format must be maintained so that compilers can save time for data input and can spend enough time for understanding of articles as well as for communication with authors.

Coded information defined in the EXFOR Exchange Format is reviewed. It is shown that the current EXFOR Exchange Format is rather flexible and can be extended to accommodate additional attributes. An example is presented for the keyword SAMPLE. There are various requests of compilation of experimental parameters (e.g., covariance matrices, neutron source spectra, resolution functions, response functions) to EXFOR entries. To archive these numerical data in EXFOR, perhaps we need to establish a general way to keep numerical data in the BIB section of the EXFOR Exchange Format. An example of response functions for a detector response function of the GELINA facility (EC-JRC IRMM) is presented to explain needs of such an extension. For compilation of covariance information, standardisation in terminology (e.g., avoiding "statistical uncertainty", "systematic uncertainty") is highly desired.

REACTION coding is one of the most difficult parts in EXFOR compilation. Compilers should not determine the REACTION code based on authors' terminology and notation, and always should go back to the original articles (especially equations), and clarify their questions with authors when necessary. Any modern format cannot simplify this process, and cannot reduce the volumes of EXFOR Compiler's Manual (LEXFOR, IAEA-NDS-208). However, I admit the current dictionary is not very friendly to explain REACTION codes to end-users. Improvement of dictionaries is desirable. Coding of complex reactions (e.g., break-up reaction) can be sometimes simplified when the branching ratio of the intermediate state to the final state is $100 \%$. In such a way, we can compile some break-up reactions within the current EXFOR Formats. Of course there are many quantities defined with complex steps and/or coordinates which cannot be simplified, and the current REACTION formalism is not very suitable for them. But it should be considered if the EXFOR users really want to find and understand the data sets with a more "advanced" REACTION formalism. Perhaps we should not develop too detailed REACTION formalism. But this does not mean we refuse compilation of such data, which can be compiled with an approximated REACTION code with a special flag "MSC" in the SF8 field if the definition of the quantity is fully described in free text, and some basic keys (e.g., target, projectile, incident energies) are kept as computer readable. One may develop an advanced EXFOR search engine -a la Google - which receive some words from users, and analyse both coded information and free text information in the EXFOR library.

\section{New formats and new features of the NDS EXFOR Web database systems (online-demo)} V. Zerkin, NDS, IAEA, Austria

\section{$\underline{\text { Topics }}$}

1. EXFOR/XML, EXFOR/html, comparison with $\mathrm{X} 4+, \mathrm{X} 4 \pm$, computational formats $\mathrm{C} 4$ and $\mathrm{C} 5$, "Standard" output as text and xml

2. EXFOR source papers: PDF-files collection, authorized access via Web retrieval system

3. EXFOR data renormalization system on the Web: automatic and based on experts' assessment

4. Remote plotting of users' data using Web-ZVView package

- Input: users data $\mathrm{y}(\mathrm{x})$ and $\mathrm{z}(\mathrm{x}, \mathrm{y})$ in various formats including non-structured data, ENDF complete files and MF33 sections, pointers to ENDF Web-archives and retrieval systems

- Actions: interactive plotting on the server side users' data including 3D animations. 
- Output: GIF, PS/EPS, PDF, copy of input data as simple columns, ENDF/MF33 files, draft of EXFOR-Covariance for compilers, data for Fortran users including reading program

5. Covariance data in EXFOR: alternative proposal for coding energy-energy correlations and reaction-reaction correlations; software support of the proposed coding in NDS Web retrieval system implemented

6. Web tools for construction covariance matrices using EXFOR uncertainties

7. Sequential search of the data missing in EXFOR, but existing in CINDA database. Import bibliographical data relevant for nuclear reaction experiments from NSR to CINDA.

8. Web uploading systems (EXFOR, ENDF, ENSDF): processing and checking of users' data by remote utilities on the server side, searching similar data and comparison (including plotting) with EXFOR/ENDF central databases

Answering some questions of the meeting participants

1. EXFOR/XML files are available from the IAEA-NDS Web retrieval system (draft version)

2. Format for coding experimental covariance data in EXFOR for reaction-reaction correlations exists, but not finalized. Supporting software of the proposed coding is already implemented under the NDS Web retrieval system

3. Web tool for construction covariance matrices using EXFOR uncertainties has been released on the IAEA-NDS and NNDC Web sites. 


\section{Meeting summaries}

EXFOR was originally (1969) created to be an "exchange format" to share data between different nuclear data centres, with different information systems and missions. By 2005, all data centres had agreed to merge all of their EXFOR libraries into one central master library and project coordination was housed in the Nuclear Data Section at the IAEA. In the mean time, the EXFOR Library has evolved into a mature and comprehensive database of knowledge transfer. Indeed, the EXFOR Library is the "Mother of All Libraries" from which nearly all evaluated nuclear application libraries (particle transport, dosimetry, etc) are derived.

Over the years, many web services have been developed to improve dissemination and data management. Currently EXFOR comprises the Format, the Information Systems (external websites, with ever-expanding retrieval and plotting capabilities, and backend data management), and the Library itself. When we discuss the future of EXFOR, we are discussing the future of all of these components of the EXFOR system and even the management of EXFOR.

The conception of EXFOR lies far in the past and despite the great foresight of its inventors and developers, new demands and impressive software developments in the past decade call for a careful evaluation of the status of EXFOR. Now is an auspicious time to do this because of the accumulated experience gained by the LLNL/BNL collaboration in the redesign and migration the ENDF format to the modern hierarchical GND format. In addition, there are many data projects around the world which would like to expand EXFOR's capabilities beyond its original design goals to accommodate new kinds of data such as the hypernuclear data currently housed in Hokkaido University's NRDF database.

We note that the EXFOR system serves many users and many communities. Our core users consist of scientists wishing to archive their data, theoreticians seeking to benchmark their models, experimentalists who seek to compare datasets, compilers who archive the data in EXFOR and evaluators who use the EXFOR data as input for their work. That said, the NRDC has limited resources and cannot compile all forms of data for all users. The compilations are guided by user needs and the extent to which the scope of archived data and format of EXFOR can be adapted to meet those needs.

The goal of this consultants meeting, was to first discuss the EXFOR Format and as a consequence the connection with the EXFOR System and Library. Along the way, we have collected users experience (especially problems) using the EXFOR Format, the EXFOR Information Systems and the EXFOR Library itself and then to present possible plans of action to address these problems. These are collected in the talk summaries and the actual presentations.

From these presentations and our collective discussions, it is our consensus strategy to keep EXFOR exchange format essentially untouched (for now). But, we have gained valuable experience from other format projects (XSAMS, ENDF/GND, ROOT, NRDF, EXFOR/XML). Several projects have proposed and even experimented with XML as an exchange mechanism for hierarchical data. This seems to be a very profitable approach and worthy of future work.

Below, we present the areas of general consensus from members of this meetings and proposals for future action items. 


\section{General consensus on extension of the EXFOR Library:}

1. Need to add incident source spectrum for average data. For example, Maxwellian Average Cross Sections are useful for validating evaluated data, yet the corresponding spectrum is not compiled in the EXFOR Library. An effort to address this need is in progress.

2. Need to store sufficient experimental data to allow a meaningful re-evaluation of experimental data. The template for the compilation of transmission factors provided by S. Kopecky et al. in the case of cadmium transmission is a good example of what is needed for the reanalysis of data.

3. Need to collect experimental response and resolution functions. The resolution is important for allowing an evaluator to match to the experimental data in EXFOR. In the cases of the Lead Slowing Down Spectrometer and measurements of double differential data and time of flight data the resolution function is quite complex. Another good example was shown by K. Kato's presentation in this meeting.

4. Need to collect experimental covariance data so evaluator can encode all correlations in their evaluations. We would like to solicit this covariance information from experimentalists. Of course, not all of the information needed to construct a proper covariance is available. So, we need a standard format for representing covariance data and supporting software.

5. Need to expand EXFOR Library with all of the associated documents for a dataset including author communication, PDFs, the experimentalist's data reduction codes, etc. This information may not be generally available outside of the EXFOR compilers due to copyright problems. Nevertheless as much as possible should be generally accessible.

\section{General consensus on the EXFOR Format:}

1. The EXFOR Format is old, but it is not necessarily "broken". The logical structure of the data hierarchy is well formed. The specific formatting of the data within the hierarchy is not very flexible. However, continued extensions are possible even if these extensions might be more convenient in another format. Currently, there is no crucial issue forcing a major change of format.

2. An editor (for compilers) and web interface (for users) insulate people who interact with the EXFOR Library from the EXFOR Format. This will allow us to modify the underlying structure/format/language of the data without interfering with compilers or users (so long as the software tools are maintained).

3. There is dissatisfaction with the restraints imposed by the EXFOR REACTION string for complex reactions. It does not easily enable coincidence data or intermediate particles (e.g. in breakup or sequential reactions). However, no consensus on how to address these shortcomings could be obtained. Work will continue to develop acceptable improvements to the REACTION coding. As an alternative we can compile this data use the existing MSC flag (the EXFOR miscellaneous flag). A poorer alternative would be to not compile this data but keep the links to the original information (e.g. through NSR).

4. There is dissatisfaction regarding the EXFOR dictionaries. Although dictionaries are essential for the EXFOR system, there are many dictionaries, they are often cryptic and the size of the dictionaries is growing. In addition, some are obsolete and there is a need for revision and possible simplification. Finally, they are hidden from the user. While most users are not interested in the contents of the dictionaries, they should be generally available.

5. Explore alternative future formats for EXFOR. There are many kinds of data that do not neatly "fit" in EXFOR. We will investigate options for alternative format, especially because projects like EXFOR/XML, GND and ROOT offer possible paths forward. 


\section{Meeting Recommendations}

Although there are many items which we arrived at a consensus regarding either the format or the library itself, we either did not agree on approaches to deal with those items or we are already dealing with them. Below we list proposals for future actions where general consensus was achieved.

1. Continue development of EXFOR/XML. Use EXFOR/XML as a standard output format since it can be easily transformed into simple to understand HTML and is isomorphic with the legacy EXFOR Format. Improve it based on user feedback and seek to harmonise it with GND. Prepare for the task of porting checking codes and other codes to a new format.

2. Improve existing EXFOR codes. Develop an editor for EXFOR/XML and improve checking codes and digitisation codes. Improve the user experience by changing the default output format of the EXFOR retrieval system.

3. Establish an "EXFOR knowledge base". This knowledge base would to integrate all the different elements of documentation in one framework, including EXFOR Formats Manual, LEXFOR Manual, EXFOR Basics Manual, Dictionary Manual, the EXFOR dictionaries (in full including explanations), Protocol, Network document and maybe even CP Memos. This knowledge base should aid the compilers but should also be available for general users.

4. Set up an "EXFOR wiki". The wiki would supplement and clarify the EXFOR manual.

5. New kinds of data. Hypernuclear reaction data are already compiled by Hokkaido U. in the NRDF database and has a large scientific interest. Some high energy p-Nucleus collision data was already compiled by Brookhaven National Laboratory, complete with needed EXFOR Format extensions, and is needed for proton radiography applications. Muon-induced reaction data are not compiled by anyone but muons comprise the dominant component of cosmic rays at sea level and therefore this data is important for understanding backgrounds in a variety of basic science and practical applications. Currently high energy data (E $>1 \mathrm{GeV} / \mathrm{A})$ compilation is voluntary, but we recommend adding already compiled data in EXFOR.

6. MSC Code in REACTION field. The current REACTION formalism cannot express some classes of experimental quantities (e.g., coincidence measurements, sequential reactions). Though we recognise some of them are useful to validate theoretical models, we recommend keeping such data with an approximate REACTION code with MSC in SF8 unless the quantities are repeatedly reported and there is an obvious need to describe the REACTION with more accuracy.

7. Governance. For such an important database it is essential that a proper set of rules and goals are laid down that are agreed by the international community and in particular the Nuclear Reaction Data Centres. A strategy document is required that establishes which user groups the library should serve, which services should be maintained and developed. Purpose, scope, quality, validation, interfaces and user-orientation are important keywords to be addressed in such a document. The EXFOR Protocol document deals with many of these issue, but is not highly advertised.

8. User feedback. Feedback from users is now a weak point and a method for a systematic approach to establishing users interest and needs should be established. Through an EXFOR user group that periodically reviews the services that are offered providing recommendations for improvements and new directions. Such a user group could meet at the upcoming ND2013 meeting where many EXFOR users will be in attendance. 
Consultant's Meeting (CM) "Further Development of EXFOR"

6 - 9 March 2012, VIC, Room A2311, IAEA, Vienna, Austria

AGENDA

Tuesday, 6 March 2012

\begin{tabular}{|c|c|}
\hline 09:00-09:30 & Registration \\
\hline 09:30-10:00 & $\begin{array}{l}\text { Welcome, Opening Remarks, and Administrative Announcements } \\
\text { Mr Robin Forrest, Section Head, NDS/IAEA - Welcome Address } \\
\text { Mr Stanislav Simakov, Meeting' Scientific Secretary, NDS/IAEA - Objectives/Outcomes of CM } \\
\text { Mr Alexander Öchs, NDS/IAEA - Meeting' administrative announcements } \\
\text { All - self introductions, selection of Chairperson \& Rapporteur, approval of Agenda }\end{array}$ \\
\hline \multicolumn{2}{|r|}{ Experiences and proposals gained from Databases (slightly) different from EXFOR } \\
\hline 10:00 - 10:30 & $\begin{array}{c}\text { Mr Bastiaan Braams, A+M Unit Head, NDS/IAEA - "Developing the XML Schema for Atoms, } \\
\text { Molecules and Solids (XSAMS)" }\end{array}$ \\
\hline $10: 30-11: 30$ & Mr Dave Brown, NNDC, Brookhaven, USA - "Thoughts on modernizing EXFOR" \\
\hline $11: 30-12: 30$ & Mr Stanislav Hlavac, IP SAS, Bratislava, Slovakia - "Updating EXFOR - necessity or luxury" \\
\hline $12: 30-14: 00$ & Lunch break \\
\hline $14: 00-15: 00$ & $\begin{array}{l}\text { Mr Kiyoshi Kato, Hokkaido University, Japan - "Comments on new EXFOR from experiences of } \\
\text { NRDF" }\end{array}$ \\
\hline $15: 00-15: 30$ & Mr Masayuki Aikawa, Hokkaido University, Japan - "New Editor and ML Format" \\
\hline $15: 30-16: 30$ & Mr Viktor Zerkin, NDS/IAEA - "XML in the IAEA-NDS EXFOR-CINDA-ENDF database systems" \\
\hline $16: 30-18: 00$ & Discussion: EXFOR and other databases - differences, similarities and mutual benefit \\
\hline
\end{tabular}

Wednesday, 7 March 2012

\begin{tabular}{|c|c|}
\hline \multicolumn{2}{|r|}{ EXFOR deficiencies and desirable improvements from end-user view } \\
\hline 9:00 - 10:00 & $\begin{array}{l}\text { Mr Arjan Plompen, EC-IRMM-JRC, Geel, Belgium - "EXFOR as a comprehensive source of } \\
\text { experimental reaction data" }\end{array}$ \\
\hline 10:00 - 11:00 & Mr Vladimir Pronyaev, IPPE, Obninsk, RF - "Coding of complex reactions in EXFOR" \\
\hline $11: 00-12: 00$ & Mr Stanislav Simakov, NDS/IAEA - "EXFOR' difficulties" \\
\hline $12: 00-13: 30$ & Lunch break \\
\hline 13:30 - 14:00 & $\begin{array}{l}\text { Ms Valentina Semkova, NDS/IAEA - "Split coding of the reaction and the measured quantity } \\
\text { possible/advantageous?" }\end{array}$ \\
\hline $14: 00-14: 30$ & Mr Dave Brown, NNDC, Brookhaven, USA - "Examples of ENDF files in gnd format" \\
\hline 14:30 - 15:00 & Discussion: Summary from EXFOR Users \\
\hline \multicolumn{2}{|r|}{ EXFOR exchange format and compilation rules - possible modifications, extensions } \\
\hline 15:00 - 16:00 & Mr Viktor Zerkin, NDS/IAEA - "EXFOR format: problems of extension and possible solutions" \\
\hline $16: 30-17: 00$ & Mr Otto Schwerer, Vienna, Austria - "EXFOR-New: Some questions, some replies" \\
\hline $17: 30-18: 30$ & $\begin{array}{c}\text { Mr Emmeric Dupont, Mr Nicolas Soppera, NEA/OECD, Paris - "Some thoughts on } \\
\text { EXchange FORmat" }\end{array}$ \\
\hline
\end{tabular}

Thursday, 8 March 2012

\begin{tabular}{|c|l|}
\hline \multicolumn{2}{|c|}{ EXFOR exchange format and compilation rules - possible modifications, extensions (cont.) } \\
\hline 9:00 - 10:00 & Mr Naohiko Otsuka, NDS/IAEA - "Comments on EXFOR from a Compiler" \\
\hline 10:00 - 10:30 & $\begin{array}{r}\text { Mr Viktor Zerkin, NDS/IAEA - "New formats and new features of the NDS EXFOR Web database } \\
\text { systems (online-demo)" }\end{array}$ \\
\hline 10:30 - 12:30 & Discussion: EXFOR exchange format - summarising and ranking of proposals \\
\hline 12:30 - 14:00 & Lunch break \\
\hline 14:00 - 18:00 & Discussion: EXFOR exchange format - summarising and ranking of proposals (cont.) \\
\hline 19:00 & Hospitality Event \\
\hline
\end{tabular}

Friday, 9 March 2012

\begin{tabular}{|l|l|}
\hline \multicolumn{2}{|c|}{ Meeting Report Drafting } \\
\hline 09:00-12:30 & Discussion and drafting of conclusions \& recommendations for Report \\
\hline 12:30-14:00 & Lunch break \\
\hline 14:00-16:00 & Final Remarks and End of the Meeting \\
\hline
\end{tabular}

Remarks: Duration for presentations includes time for questions, Coffee breaks - as needed 


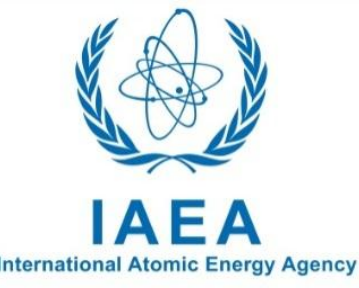

Atoms for Peace

Consultants meeting on

"Further Development of EXFOR"

6-9 March 2012, Vienna International Centre, Meeting room A-2311

Vienna, Austria

\section{LIST OF PARTICIPANTS}

\begin{tabular}{|c|c|}
\hline AUSTRIA & BELGIUM \\
\hline 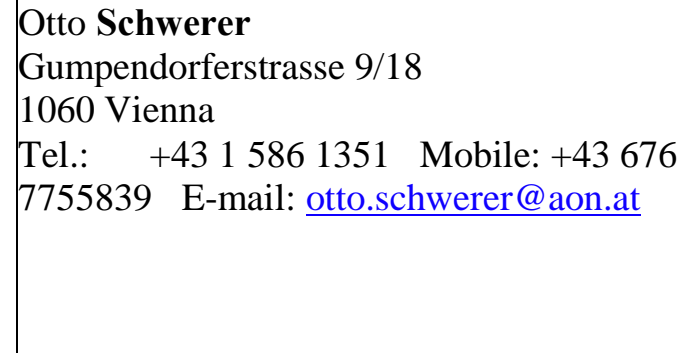 & $\begin{array}{l}\text { Arjan Plompen } \\
\text { European Commission-JRC-IRMM } \\
\text { Nuclear Physics Unit } \\
\text { Retieseweg } 111 \\
\text { 2440 Geel } \\
\text { Tel.: +32 } 14571381 \\
\text { Fax: +32 } 14571376 \\
\text { E-mail: Arjan.Plompen@ec.europa.eu }\end{array}$ \\
\hline JAPAN & JAPAN \\
\hline $\begin{array}{l}\text { Masayuki Aikawa } \\
\text { Faculty of Science } \\
\text { Hokkaido University } \\
\text { Kita } 10 \text { Nishi 8, Kita-ku } \\
\text { Sapporo 060-0810 } \\
\text { Tel.: +81 } 117063723 \\
\text { Fax: +81 } 117063724 \\
\text { E-mail: aikawa@ @ nucl.sci.hokudai.ac.jp }\end{array}$ & $\begin{array}{l}\text { Kiyoshi Kato } \\
\text { Department of Physics } \\
\text { Hokkaido University } \\
\text { Kita-10, Nishi-8 Kita-ku } \\
\text { Sapporo 060-0810 } \\
\text { Tel.: +81 } 117062684 \\
\text { Fax: +81 } 11 \text { 7062684 } \\
\text { E-mail: kato@ nucl.sci.hokudai.ac.jp }\end{array}$ \\
\hline RUSSIAN FEDERATION & SLOVAKIA \\
\hline $\begin{array}{l}\text { Vladimir Pronyaev } \\
\text { Nuclear Data Centre } \\
\text { Institute of Physics and Power Engineering } \\
\text { Bondarenko sq. } 1 \\
249033 \text { Obninsk, Kaluga Region } \\
\text { Tel.: +7 48439-94589 } \\
\text { Fax: +7 48439-76083 } \\
\text { E-mail: pronyaev@ippe.ru }\end{array}$ & $\begin{array}{l}\text { Stanislav Hlavac } \\
\text { Department of Nuclear Physics } \\
\text { Institute of Physics SAS } \\
\text { Dubravska cesta } 9 \\
84511 \text { Bratislava } 45 \\
\text { Tel.: +421 } 259410500 \\
\text { Fax: +421 } 269201900 \\
\text { E-mail: hlavac@ @avba.sk }\end{array}$ \\
\hline
\end{tabular}




\begin{tabular}{|c|c|}
\hline USA & OECD \\
\hline $\begin{array}{l}\text { David Brown } \\
\text { Brookhaven National Laboratory } \\
\text { Building 197D } \\
\text { Upton NY 11973-5000 } \\
\text { Tel.: +1 631 344-2814 } \\
\text { Fax: +1 631 344-2806 } \\
\text { E-mail: dbrown@ bnl.gov }\end{array}$ & $\begin{array}{l}\text { Emmeric Dupont } \\
\text { OECD Nuclear Energy Agency Data Bank } \\
\text { 12, Boulevard des Iles } \\
\text { F-92130 Issy-Les-Moulineaux } \\
\text { Tel.: +33 } 145241084 \\
\text { Fax: +33 } 1452411 \text { 10 } \\
\text { E-mail: emmeric.dupont@oecd.org }\end{array}$ \\
\hline OECD & IAEA \\
\hline $\begin{array}{l}\text { Nicolas Soppera } \\
\text { OECD Nuclear Energy Agency Data Bank } \\
\text { 12, Boulevard des Iles } \\
\text { F-92130 Issy-Les-Moulineaux } \\
\text { Tel.: +33 } 145241088 \\
\text { Fax: +33 } 145241110 \\
\text { E-mail: nicolas.soppera@ oecd.org }\end{array}$ & $\begin{array}{l}\text { Robin A. Forrest } \\
\text { Head, Nuclear Data Section } \\
\text { Division of Physical and Chemical Sciences } \\
\text { Tel.: +43 } 1260021709 \\
\text { Fax: +43 } 12600721709 \\
\text { E-mail: r.forrest@ @iaea.org }\end{array}$ \\
\hline IAEA & IAEA \\
\hline $\begin{array}{l}\text { Stanislav Simakov } \\
\text { Head, Nuclear Data Services Unit } \\
\text { Nuclear Data Section } \\
\text { Division of Physical and Chemical Sciences } \\
\text { Tel.: +43 } 1260021717 \\
\text { Fax: +43 } 12600721717 \\
\text { E-mail: s.simakov@iaea.org }\end{array}$ & $\begin{array}{l}\text { Naohiko Otsuka } \\
\text { Nuclear Data Section } \\
\text { Division of Physical and Chemical Sciences } \\
\text { Tel.: +43 } 1260021715 \\
\text { Fax: +43 } 12600721715 \\
\text { E-mail: n.otsuka@ @iaea.org }\end{array}$ \\
\hline IAEA & IAEA \\
\hline $\begin{array}{l}\text { Valentina Semkova } \\
\text { Nuclear Data Section } \\
\text { Division of Physical and Chemical Sciences } \\
\text { Tel.: +43 } 1260021727 \\
\text { Fax: +43 } 12600721727 \\
\text { E-mail: v.semkova@iaea.org }\end{array}$ & $\begin{array}{l}\text { Viktor Zerkin } \\
\text { Nuclear Data Section } \\
\text { Division of Physical and Chemical Sciences } \\
\text { Tel.: +43 } 1260021714 \\
\text { Fax: +43 } 12600721714 \\
\text { E-mail: } \text { v.zerkin@ iaea.org }\end{array}$ \\
\hline IAEA & IAEA \\
\hline $\begin{array}{l}\text { Bastian Braams Head, Atomic and Molecular } \\
\text { Data Unit Nuclear Data Section Division of } \\
\text { Physical and Chemical Sciences Tel.: +43 } 12600 \\
21727 \text { Fax: +43 } 12600721727 \text { E-mail: } \\
\text { v.semkova@iaea.org }\end{array}$ & $\begin{array}{l}\text { Roberto Capote Noy } \\
\text { Nuclear Data Section } \\
\text { Division of Physical and Chemical Sciences } \\
\text { Tel.: +43 } 1260021713 \\
\text { Fax: +43 } 12600721714 \\
\text { E-mail: v.zerkin@ iaea.org }\end{array}$ \\
\hline
\end{tabular}



Nuclear Data Section

International Atomic Energy Agency

Vienna International Centre, P.O. Box 100

A-1400 Vienna

Austria e-mail: services@iaeand.iaea.org fax: (43-1) 26007

telephone: (43-1) 2600-21710

Web: http://www-nds.iaea.org 